A. F DIL (Marrakech)

\title{
SOME RESULTS ON CONVERGENCE ACCELERATION FOR THE E-ALGORITHM
}

Abstract. Some new results on convergence acceleration for the $E$-algorithm which is a general extrapolation method are obtained. A technique for avoiding numerical instability is proposed. Some applications are given. Theoretical results are illustrated by numerical experiments.

1. Introduction. The $E$-algorithm [2], [7] is a general extrapolation process. It depends on some auxiliary sequences $\left(g_{i}(n)\right), i \geq 1$. For some choices of the auxiliary sequences, one obtains some known convergence acceleration methods (see [2], [3]). When applied to a sequence $\left(s_{n}\right)$ of complex numbers, the algorithm has the following rules:

$$
\begin{array}{ll}
E_{0}^{(n)}=s_{n}, \quad g_{0, i}^{(n)}=g_{i}(n), & n \geq 0, i \geq 1, \\
E_{k}^{(n)}=\frac{g_{k-1, k}^{(n+1)} E_{k-1}^{(n)}-g_{k-1, k}^{(n)} E_{k-1}^{(n+1)}}{g_{k-1, k}^{(n+1)}-g_{k-1, k}^{(n)}}, & k \geq 1 \text { (main rule), } \\
g_{k, j}^{(n)}=\frac{g_{k-1, k}^{(n+1)} g_{k-1, j}^{(n)}-g_{k-1, k}^{(n)} g_{k-1, j}^{(n+1)}}{g_{k-1, k}^{(n+1)}-g_{k-1, k}^{(n)}}, & j>k \text { (auxiliary rule). }
\end{array}
$$

Some results on convergence acceleration for the $E$-algorithm were obtained by Brezinski [2] when the auxiliary sequences satisfy the following condition:

$$
\forall i \geq 1, \quad \frac{g_{i}(n+1)}{g_{i}(n)} \underset{n \rightarrow \infty}{\longrightarrow} b_{i} \neq 1, \quad \text { and } \quad b_{i} \neq b_{j} \text { for } i \neq j .
$$

This condition will be called Brezinski's condition.

1991 Mathematics Subject Classification: Primary 65B05.

Key words and phrases: convergence acceleration, extrapolation, summation of series, numerical quadrature. 
In this article, we give some results on convergence acceleration in three cases where Brezinski's condition is not satisfied.

All the sequences considered are sequences of real numbers.

In Section 1, we consider the case where $g_{i}(n+1)=g(n) b_{i}^{n}$ for all $i \geq 1$ with $g(n+1) / g(n) \rightarrow 0$ as $n \rightarrow \infty$. Since $g_{i}(n+1) / g_{i}(n) \rightarrow 0$ as $n \rightarrow \infty$ for all $i \geq 1$, we cannot apply the results obtained by Brezinski [2].

In Section 2, we study the $E$-algorithm when the auxiliary sequences are such that for each $i \geq 1, g_{i}(n)$ has an asymptotic expansion of the form

$$
g_{i}(n) \approx \lambda_{i}^{n} n^{\theta_{i}}\left(a_{i, 0}+\frac{a_{i, 1}}{n^{\alpha_{i, 1}}}+\ldots+\frac{a_{i, j}}{n^{\alpha_{i, j}}}+\ldots\right) .
$$

When the numbers $\lambda_{i}$ are close to 1 , the $E$-algorithm is numerically unstable. In order to avoid numerical instability, we propose to apply the $E$-algorithm with some subsequences of $\left(g_{i}(n)\right)$ as auxiliary sequences. This technique is very interesting in particular when $\left(s_{n}\right)$ has the following asymptotic expansion:

$$
s_{n}-s \approx a_{1} g_{1}(n)+a_{2} g_{2}(n)+\ldots+a_{i} g_{i}(n)+\ldots
$$

By choosing appropriately the subsequences of the auxiliary sequences, we can apply the results of Section 1 where the auxiliary sequences converge superlinearly to 0 . This technique is illustrated by numerical examples in Section 3.

Section 3 is devoted to the application of the $E$-algorithm to the summation of series and computation of integrals.

2. Superlinear convergence. Let us begin by the following notations and definitions. $\mathbb{N}$ denotes the set of positive integers, and $\mathbb{R}$ the set of real numbers. If $\left(s_{n}\right)$ is a convergent sequence, we denote by $s$ its limit; $u_{n}=o\left(v_{n}\right)$ means that $u_{n} / v_{n} \rightarrow 0$ as $n \rightarrow \infty$.

Definitions. Let $\left(f_{1}(n)\right),\left(f_{2}(n)\right), \ldots,\left(f_{i}(n)\right), \ldots$ be some sequences of real numbers.

- We say that $\left(f_{1}, f_{2}, \ldots, f_{i}, \ldots\right)$ is an asymptotic sequence if for each index $i, f_{i+1}(n)=o\left(f_{i}(n)\right)$ as $n \rightarrow \infty$.

Let $\left(u_{n}\right)$ be a sequence of real numbers, and $\left(f_{1}, f_{2}, \ldots, f_{i}, \ldots\right)$ be an asymptotic sequence.

- We say that $\left(u_{n}\right)$ has an asymptotic expansion with respect to the asymptotic sequence $\left(f_{1}, f_{2}, \ldots, f_{i}, \ldots\right)$ if there exist constants $a_{i} \in \mathbb{R}, i \geq 1$, such that for all $k \geq 1$,

$$
u_{n}=\sum_{i=1}^{k} a_{i} g_{j}(n)+o\left(g_{k}(n)\right) \quad \text { as } n \rightarrow \infty \text {. }
$$


Then we write

$$
u_{n} \approx a_{1} g_{1}(n)+\ldots+a_{k} g_{k}(n)+\ldots
$$

Definitions. Let $A$ be a set of convergent sequences.

- We say that the $E$-algorithm is regular on $A$ if for all $\left(s_{n}\right) \in A$ and $k \geq 1$,

$$
E_{k}^{(n)}-s=o(1) \quad \text { as } n \rightarrow \infty .
$$

- We say that the $E$-algorithm is effective on $A$ if for all $\left(s_{n}\right) \in A$ and $k \geq 1$, either

$\exists n_{0}, \forall n \geq n_{0}, E_{k}^{(n)}=E_{k-1}^{(n)}=s \quad$ or $\quad E_{k}^{(n)}-s=o\left(E_{k-1}^{(n)}-s\right)$ as $n \rightarrow \infty$.

Let us now assume that the auxiliary sequences of the $E$-algorithm are such that

$\left(\mathrm{H}_{1}\right) \quad \forall i \geq 1, g_{i}(n)=g(n) b_{i}^{n}$, where $g(n+1)=o(g(n)), b_{1}=1$, and $b_{i} \neq b_{j}$ for $i \neq j$.

Then Brezinski's condition is not satisfied.

Lemma 1. For any $k \geq 0$ and $i>k$, we have

$$
\begin{aligned}
& \forall n, \quad \frac{g_{k, i}^{(n+1)}}{g_{k, i}^{(n)}}=\frac{b_{i}}{b_{k+1}} \cdot \frac{g_{k, k+1}^{(n+1)}}{g_{k, k+1}^{(n)}} ; \\
& g_{k, i}^{(n+1)}=o\left(g_{k, i}^{(n)}\right) \quad \text { as } n \rightarrow \infty .
\end{aligned}
$$

Proof. By induction on $k$.

THEOREM 1. Let $\left(s_{n}\right)$ be a convergent sequence. If the auxiliary sequences $\left(g_{i}(n)\right)$ of the E-algorithm satisfy $\left(\mathrm{H}_{1}\right)$, then $E_{k}^{(n)} \rightarrow s$ as $n \rightarrow \infty$, for each $k \geq 0$.

Pr o of (induction on $k$ ). For $k=0$, the result is obvious. Assume that

$$
E_{k}^{(n)}-s=o(1) \quad \text { as } n \rightarrow \infty .
$$

From the main rule of the $E$-algorithm, we get

$$
E_{k+1}^{(n)}-s=\frac{\frac{g_{k, k+1}^{(n+1)}}{g_{k, k+1}^{(n)}}\left(E_{k}^{(n)}-s\right)-\left(E_{k}^{(n+1)}-s\right)}{\frac{g_{k, k+1}^{(n+1)}}{g_{k, k+1}^{(n)}}-1}
$$

From Lemma 1 we get

$$
g_{k, k+1}^{(n+1)}=o\left(g_{k, k+1}^{(n)}\right) \quad \text { as } n \rightarrow \infty .
$$

From (1)-(3) we deduce that $E_{k+1}^{(n)}-s=o(1)$ as $n \rightarrow \infty$. 
THEOREM 2. Let $k \geq 1$. If the conditions of Theorem 1 are satisfied, then

(i) $E_{k}^{(n)}-s=o\left(E_{k-1}^{(n)}-s\right)$ as $n \rightarrow \infty$ iff $E_{k-1}^{(n+1)}-s=o\left(E_{k-1}^{(n)}-s\right)$ as $n \rightarrow \infty$;

(ii) $E_{k}^{(n)}-s=o\left(E_{k-1}^{(n+1)}-s\right)$ as $n \rightarrow \infty$ iff $\frac{\left(E_{k-1}^{(n+1)}-s\right) g_{k-1, k}^{(n)}}{\left(E_{k-1}^{(n)}-s\right) g_{k-1, k}^{(n+1)}} \underset{n \rightarrow \infty}{\longrightarrow} 1$.

Proof. (i) We have

$$
\frac{E_{k}^{(n)}-s}{E_{k-1}^{(n)}-s}=\frac{\frac{g_{k-1, k}^{(n+1)}}{g_{k-1, k}^{(n)}}-\frac{E_{k-1}^{(n+1)}-s}{E_{k-1}^{(n)}-s}}{\frac{g_{k-1, k}^{(n+1)}}{g_{k-1, k}^{(n)}}-1} .
$$

Lemma 1(ii) gives

$$
g_{k-1, k}^{(n+1)}=o\left(g_{k-1, k}^{(n)}\right) \quad \text { as } n \rightarrow \infty .
$$

From (4), (5) we deduce that

$E_{k}^{(n)}-s=o\left(E_{k-1}^{(n)}-s\right)$ as $n \rightarrow \infty \Leftrightarrow E_{k-1}^{(n+1)}-s=o\left(E_{k-1}^{(n)}-s\right)$ as $n \rightarrow \infty$.

(ii) We have

$$
\frac{E_{k}^{(n)}-s}{E_{k-1}^{(n+1)}-s}=\frac{\frac{g_{k-1, k}^{(n+1)}}{g_{k-1, k}^{(n)}} \cdot \frac{E_{k-1}^{(n)}-s}{E_{k-1}^{(n+1)}-s}-1}{\frac{g_{k-1, k}^{(n+1)}}{g_{k-1, k}^{(n)}}-1} .
$$

From (5), (6) we get

$$
E_{k}^{(n)}-s=o\left(E_{k-1}^{(n+1)}-s\right) \text { as } n \rightarrow \infty \Leftrightarrow \frac{\left(E_{k-1}^{(n+1)}-s\right) g_{k-1, k}^{(n)}}{\left(E_{k-1}^{(n)}-s\right) g_{k-1, k}^{(n+1)}} \underset{n \rightarrow \infty}{\longrightarrow} 1 .
$$

PROPERTy 1. If the auxiliary sequences $\left(g_{i}(n)\right)$ satisfy $\left(\mathrm{H}_{1}\right)$ with $\left|b_{i+1}\right|<$ $\left|b_{i}\right|$ for $i \geq 1$, then for $k \geq 0$ and $i>k$,

$$
g_{k, i+1}^{(n)}=o\left(g_{k, i}^{(n)}\right) \quad \text { as } n \rightarrow \infty
$$

(i.e. $\left(g_{k, k+1}, g_{k, k+2}, \ldots, g_{k, i}, \ldots\right)$ is an asymptotic sequence).

P r o of. From Lemma 1 we get

$$
\frac{g_{k, i}^{(n+1)}}{g_{k, i}^{(n)}}=\frac{b_{i}}{b_{k+1}} \cdot \frac{g_{k, k+1}^{(n+1)}}{g_{k, k+1}^{(n)}}, \quad \frac{g_{k, i+1}^{(n+1)}}{g_{k, i+1}^{(n)}}=\frac{b_{i+1}}{b_{k+1}} \cdot \frac{g_{k, k+1}^{(n+1)}}{g_{k, k+1}^{(n)}} .
$$


Thus

Consequently,

$$
\frac{g_{k, i+1}^{(n+1)}}{g_{k, i+1}^{(n)}}=\frac{b_{i+1}}{b_{i}} \cdot \frac{g_{k, i}^{(n+1)}}{g_{k, i}^{(n)}} .
$$

$$
\frac{g_{k, i+1}^{(n)}}{g_{k, i}^{(n)}}=\left(\frac{b_{i+1}}{b_{i}}\right)^{n} \frac{g_{k, i+1}^{(0)}}{g_{k, i}^{(0)}} .
$$

Since $\left|b_{i+1}\right|<\left|b_{i}\right|$, it follows that $g_{k, i+1}^{(n)}=o\left(g_{k, i}^{(n)}\right)$ as $n \rightarrow \infty$.

Let us mention that, if the auxiliary sequences $\left(g_{i}(n)\right)$ of the $E$-algorithm form an asymptotic sequence, then in general, for $k \geq 1,\left(g_{k, k+1}, g_{k, k+2}\right.$, $\left.\ldots, g_{k, i}, \ldots\right)$ is not an asymptotic sequence. For example, let

$$
g_{i}(n)=(-1)^{n(i+1)} /(n+1)^{i}, \quad n \geq 0, i \geq 1 .
$$

Then $\left(g_{1}, g_{2}, \ldots, g_{i}, \ldots\right)$ is an asymptotic sequence, but $\left(g_{1,2}, g_{1,3}, \ldots\right.$ $\left.\ldots, g_{1, i}, \ldots\right)$ is not.

LeMma 2. Let $\left(s_{n}\right)$ be a convergent sequence. If

(i) the auxiliary sequences $\left(g_{i}(n)\right)$ satisfy $\left(\mathrm{H}_{1}\right)$ with $\left|b_{i+1}\right|<\left|b_{i}\right|$ for $i \geq 1$,

(ii) $s_{n}-s \approx a_{1} g_{1}(n)+a_{2} g_{2}(n)+\ldots+a_{i} g_{i}(n)+\ldots$,

then for each $k \geq 0, E_{k}^{(n)}-s \approx a_{k+1} g_{k, k+1}^{(n)}+\ldots+a_{i} g_{k, i}^{(n)}+\ldots$

Proof. By induction on $k$.

Remark. If $a_{j}=0$ for all $j>k$, then there exists $n_{0}$ such that $E_{k}^{(n)}=s$ for $n \geq n_{0}$; this result is a particular case of a general result given by Brezinski [2] for the kernel of step $k$ of the $E$-algorithm.

THEOREM 3. Let $\left(s_{n}\right)$ be a convergent sequence. If the auxiliary sequences $\left(g_{i}(n)\right)$ satisfy $\left(\mathrm{H}_{1}\right)$ with $\left|b_{i+1}\right|<\left|b_{i}\right|$ for $i \geq 1$, and if

$$
s_{n}-s \approx a_{1} g_{1}(n)+a_{2} g_{2}(n)+\ldots+a_{i} g_{i}(n)+\ldots,
$$

then for all $k \geq 1$,

(i) if $a_{i}=0$ for all $i>k$, then there exists $n_{0}$ such that $E_{k}^{(n)}=s$ for all $n \geq n_{0}$

(ii) if $a_{k} \neq 0$, then $E_{k}^{(n)}-s=o\left(E_{k-1}^{(n+1)}-s\right)$ as $n \rightarrow \infty$;

(iii) if $a_{k}=0$ and there exists $i>k$ such that $a_{i} \neq 0$, then

$$
\begin{aligned}
& E_{k}^{(n)}-s=o\left(E_{k-1}^{(n)}-s\right) \quad \text { as } n \rightarrow \infty, \\
& E_{k}^{(n)}-s \neq o\left(E_{k-1}^{(n+1)}-s\right) \quad \text { as } n \rightarrow \infty .
\end{aligned}
$$

Proof. (i) follows from the preceding remark. 
(ii) Assume that $a_{k} \neq 0$. From Lemma 2, we get $E_{k-1}^{(n)}-s=a_{k} g_{k-1, k}^{(n)}+$ $o\left(g_{k-1, k}^{(n)}\right)$ as $n \rightarrow \infty$. Thus

$$
\frac{\left(E_{k-1}^{(n+1)}-s\right) g_{k-1, k}^{(n)}}{\left(E_{k-1}^{(n)}-s\right) g_{k-1, k}^{(n+1)}} \underset{n \rightarrow \infty}{\longrightarrow} 1
$$

and from Theorem 2 we deduce that $E_{k}^{(n)}-s=o\left(E_{k-1}^{(n+1)}-s\right)$ as $n \rightarrow \infty$.

(iii) Let $i_{0}>k$ be the smallest integer such that $a_{i_{0}} \neq 0$. From Lemma 2 we get $E_{k-1}^{(n)}-s=a_{i_{0}} g_{k-1, i_{0}}^{(n)}+o\left(g_{k-1, i_{0}}^{(n)}\right)$ as $n \rightarrow \infty$. Thus

$$
\frac{E_{k-1}^{(n+1)}-s}{E_{k-1}^{(n)}-s}=\frac{g_{k-1, i_{0}}^{(n+1)}}{g_{k-1, i_{0}}^{(n)}}(1+o(1)) \quad \text { as } n \rightarrow \infty .
$$

From Lemma 1, we get

$$
g_{k-1, i_{0}}^{(n+1)}=o\left(g_{k-1, i_{0}}^{(n)}\right) \quad \text { as } n \rightarrow \infty .
$$

The relations $(7),(8)$ and Theorem $2(\mathrm{i})$ give $E_{k}^{(n)}-s=o\left(E_{k-1}^{(n)}-s\right)$ as $n \rightarrow \infty$.

We have

$$
\frac{\left(E_{k-1}^{(n+1)}-s\right) g_{k-1, k}^{(n)}}{\left(E_{k-1}^{(n)}-s\right) g_{k-1, k}^{(n+1)}}=\frac{g_{k-1, i_{0}}^{(n+1)}}{g_{k-1, i_{0}}^{(n)}} \cdot \frac{g_{k-1, k}^{(n)}}{g_{k-1, k}^{(n+1)}}(1+o(1)) \quad \text { as } n \rightarrow \infty .
$$

From Lemma 1, we deduce that

$$
\frac{g_{k-1, i_{0}}^{(n+1)}}{g_{k-1, i_{0}}^{(n)}} \cdot \frac{g_{k-1, k}^{(n)}}{g_{k-1, k}^{(n+1)}}=\frac{b_{i_{0}}}{b_{k}}
$$

The relations (9), (10) show that the sequence

$$
\left(E_{k-1}^{(n+1)}-s\right) g_{k-1, k}^{(n)} /\left(\left(E_{k-1}^{(n)}-s\right) g_{k-1, k}^{(n+1)}\right)
$$

does not converge to 1 ; then, from Theorem 2 , we deduce that $E_{k}^{(n)}-s \neq$ $o\left(E_{k-1}^{(n+1)}-s\right)$ as $n \rightarrow \infty$.

Let us now assume that the sequence $\left(s_{n}\right)$ satisfies

$$
s_{n}-s \approx g(n)\left(a_{1} c_{1}^{n}+a_{2} c_{2}^{n}+\ldots+a_{i} c_{i}^{n}+\ldots\right),
$$

where $a_{1} \neq 0,\left|c_{1}\right|>\ldots>\left|c_{n}\right|>\left|c_{n+1}\right|>\ldots>0$. Let $\left(r_{j, i}^{(n)}\right), j \geq 0$, $i \geq 1$, be the sequences obtained by applying the $E$-algorithm with the auxiliary sequences $g_{k}(n)=g(n) b_{k}^{n}$ to the sequence $\left(g(n) c_{i}^{n}\right)$. We have

$$
\begin{array}{ll}
r_{0, i}^{(n)}=g(n) c_{i}^{n}, & n \geq 0, \\
r_{j, i}^{(n)}=\frac{g_{j-1, j}^{(n+1)} r_{j-1, i}^{(n)}-g_{j-1, j}^{(n)} r_{j-1, i}^{(n+1)}}{g_{j-1, j}^{(n+1)}-g_{j-1, j}^{(n)}}, & j \geq 1 .
\end{array}
$$


By induction on $k$, one can easily prove the following lemmas.

Lemma 3. Let $i \geq 1$. Then

$$
\forall k \geq 0, \forall j>k, \forall n \geq 0, \quad \frac{r_{k, i}^{(n+1)}}{r_{k, i}^{(n)}}=\frac{c_{i}}{b_{j}} \cdot \frac{g_{k, j}^{(n+1)}}{g_{k, j}^{(n)}} .
$$

LEMMA 4. If the auxiliary sequences $\left(g_{i}(n)\right)$ satisfy $\left(\mathrm{H}_{1}\right)$, and if $\left(s_{n}\right)$ satisfies (11), then for all $k \geq 0$,

$$
E_{k}^{(n)}-s \approx a_{1} r_{k, 1}^{(n)}+a_{2} r_{k, 2}^{(n)}+\ldots+a_{i} r_{k, i}^{(n)}+\ldots
$$

THEOREM 4. If the conditions of Lemma 4 are satisfied, then the E-algorithm is effective on $\left(s_{n}\right)$.

Proof. Let $k \geq 0$. Lemma 4 gives

$$
E_{k}^{(n)}-s \approx a_{1} r_{k, 1}^{(n)}+\ldots+a_{i} r_{k, i}^{(n)}+\ldots
$$

From Lemma 3, we get

$$
\frac{r_{k, 1}^{(n+1)}}{r_{k, 1}^{(n)}}=\frac{c_{1}}{b_{k+1}} \cdot \frac{g_{k, k+1}^{(n+1)}}{g_{k, k+1}^{(n)}}
$$

Then, from Lemma 1, we obtain

$$
r_{k, 1}^{(n+1)}=o\left(r_{k, 1}^{(n)}\right) \quad \text { as } n \rightarrow \infty .
$$

From (12), (13) we deduce that $E_{k}^{(n+1)}-s=o\left(E_{k}^{(n)}-s\right)$ as $n \rightarrow \infty$, and from Theorem 2 we obtain $E_{k+1}^{(n)}-s=o\left(E_{k}^{(n)}-s\right)$ as $n \rightarrow \infty$. Consequently, the $E$-algorithm is effective on $\left(s_{n}\right)$.

2. Linear and logarithmic convergence. In this section, we shall study the $E$-algorithm when the auxiliary sequences are such that

$\left(\mathrm{H}_{2}\right) \quad \forall i \geq 1, \quad g_{i}(n) \approx \lambda_{i}^{n} n^{\theta_{i}}\left(a_{i, 0}+\frac{a_{i, 1}}{n^{\alpha_{i, 1}}}+\ldots+\frac{a_{i, j}}{n^{\alpha_{i, j}}}+\ldots\right)$,

where $a_{i, 0} \neq 0,0<\alpha_{i, 1}<\alpha_{i, 2}<\ldots<\alpha_{i, j}<\ldots$, and $\left(\lambda_{i}, \theta_{i}\right) \neq\left(\lambda_{j}, \theta_{j}\right)$ for $i \neq j$.

Remark. If $\lambda_{i} \neq \lambda_{j}$ for $i \neq j$, then Brezinski's condition is satisfied.

2.1. Linear convergence. From the sequence $\left(\theta_{i}\right)$, we define the following double sequence:

and for $k \geq 1$ and $i>k$,

$$
\theta_{0, i}=\theta_{i} \quad \text { for } i \geq 1,
$$

$$
\theta_{k, i}= \begin{cases}\theta_{k-1, i}-1 & \text { if } \lambda_{i}=\lambda_{k} \\ \theta_{k-1, i} & \text { else. }\end{cases}
$$


One can easily prove the following property:

Property 2. Let $k \geq 0$. Let $j>i>k$. If $\lambda_{i}=\lambda_{j}$, then

(i) $\theta_{k, i} \neq \theta_{k, j}$;

(ii) if $\theta_{j}<\theta_{i}$, then $\theta_{k, j}<\theta_{k, i}$.

Property 3. If the auxiliary sequences $\left(g_{i}(n)\right)$ satisfy $\left(\mathrm{H}_{2}\right)$ with $\lambda_{i} \neq 1$ for $i \geq 1$, then for all $k \geq 0$ and $i>k$,

$$
g_{k, i}^{(n)} \approx \lambda_{i}^{n} n^{\theta_{k, i}}\left(a_{k, i, 0}+\frac{a_{k, i, 1}}{n^{\alpha_{k, i, 1}}}+\ldots+\frac{a_{k, i, j}}{n^{\alpha_{k, i, j}}}+\ldots\right)
$$

where $a_{k, i, 0} \neq 0$ and $0<\alpha_{k, i, 1}<\ldots<\alpha_{k, i, j}<\ldots$

Proof (induction on $k$ ). For $k=0$, the property is true. Assume that it is true up to index $k$. Let $i>k+1$. From the auxiliary rule of the $E$-algorithm we get

$$
\frac{g_{k+1, i}^{(n)}}{g_{k, i}^{(n)}}=\frac{\frac{g_{k, k+1}^{(n+1)}}{g_{k, k+1}^{(n)}}-\frac{g_{k, i}^{(n+1)}}{g_{k, i}^{(n)}}}{\frac{g_{k, k+1}^{(n+1)}}{g_{k, k+1}^{(n)}}-1} .
$$

From the induction assumption we get

$$
g_{k, i}^{(n)} \approx \lambda_{i}^{n} n^{\theta_{k, i}}\left(a_{k, i, 0}+\frac{a_{k, i, 1}}{n^{\alpha_{k, i, 1}}}+\ldots+\frac{a_{k, i, j}}{n^{\alpha_{k, i, j}}}+\ldots\right),
$$

where $a_{k, i, 0} \neq 0$ and $0<\alpha_{k, i, 1}<\ldots<\alpha_{k, i, j}<\ldots$, and

$$
g_{k, k+1}^{(n)} \approx \lambda_{k+1}^{n} n^{\theta_{k, k+1}}\left(a_{k, k+1,0}+\frac{a_{k, k+1,1}}{n^{\alpha_{k, k+1,1}}}+\ldots+\frac{a_{k, k+1, j}}{n^{\alpha_{k, k+1, j}}}+\ldots\right),
$$

where $a_{k, k+1,0} \neq 0$ and $0<\alpha_{k, k+1,1}<\ldots<\alpha_{k, k+1, j}<\ldots$ Thus

$$
\frac{g_{k, i}^{(n+1)}}{g_{k, i}^{(n)}} \approx \lambda_{i}\left(1+\frac{\theta_{k, i}}{n}+\frac{d_{i, 1}}{n^{p_{i, 1}}}+\ldots+\frac{d_{i, j}}{n^{p_{i, j}}}+\ldots\right),
$$

where $1<p_{i, 1}<\ldots<p_{i, j}<\ldots$, and

$$
\frac{g_{k, k+1}^{(n+1)}}{g_{k, k+1}^{(n)}} \approx \lambda_{k+1}\left(1+\frac{\theta_{k, k+1}}{n}+\frac{d_{k+1,1}}{n^{p_{k+1,1}}}+\ldots+\frac{d_{k+1, j}}{n^{p_{k+1, j}}}+\ldots\right),
$$

where $1<p_{k+1,1}<\ldots<p_{k+1, j}<\ldots$ From (14), (17), (18) we get

$$
\frac{g_{k+1, i}^{(n)}}{g_{k, i}^{(n)}} \approx \frac{\left(\lambda_{k+1}-\lambda_{i}\right)+\frac{\lambda_{k+1} \theta_{k, k+1}-\lambda_{i} \theta_{k, i}}{n}+\ldots}{\lambda_{k+1}-1+\frac{\lambda_{k+1} \theta_{k, k+1}}{n}+\ldots} .
$$


If $\lambda_{i} \neq \lambda_{k+1}$, then the relations (15), (19) give

$$
g_{k+1, i}^{(n)} \approx \lambda_{i}^{n} n^{\theta_{k+1, i}}\left(a_{k+1, i, 0}+\frac{a_{k+1, i, 1}}{n^{\alpha_{k+1, i, 1}}}+\ldots+\frac{a_{k+1, i, j}}{n^{\alpha_{k+1, i, j}}}+\ldots\right)
$$

with $\theta_{k+1, i}=\theta_{k, i}$,

$$
a_{k+1, i, 0}=\frac{a_{k, i, 0}\left(\lambda_{k+1}-\lambda_{i}\right)}{\lambda_{k+1}-1} \neq 0,
$$

and $0<\alpha_{k+1, i, 1}<\ldots<\alpha_{k+1, i, j}<\ldots$

If $\lambda_{i}=\lambda_{k+1}$, then, from Property 2 , we get $\theta_{k, i} \neq \theta_{k, k+1}$, and from (15), (19) we deduce that

$$
g_{k+1, i}^{(n)} \approx \lambda_{i}^{n} n^{\theta_{k+1, i}}\left(a_{k+1, i, 0}+\frac{a_{k+1, i, 1}}{n^{\alpha_{k+1, i, 1}}}+\ldots+\frac{a_{k+1, i, j}}{n^{\alpha_{k+1, i, j}}}+\ldots\right)
$$

with $\theta_{k+1, i}=\theta_{k, i}-1$,

$$
a_{k+1, i, 0}=\frac{a_{k, i, 0}\left(\theta_{k, k+1}-\theta_{k, i}\right) \lambda_{k+1}}{\lambda_{k+1}-1} \neq 0,
$$

and $0<\alpha_{k+1, i, 1}<\ldots<\alpha_{k+1, i, j}<\ldots$

Thus the property is true for $k+1$.

Property 4. If the condition of Property 3 is satisfied, then for all $k \geq 0$ and $i>k$,

Proof. This is obvious.

$$
g_{k, i}^{(n+1)} / g_{k, i}^{(n)} \underset{n \rightarrow \infty}{\longrightarrow} \lambda_{i}
$$

An immediate consequence of Property 4 is

TheOREm 5. Let $\left(s_{n}\right)$ be a convergent sequence. If the condition of Property 3 is satisfied, then for all $k \geq 0, E_{k}^{(n)} \rightarrow s$ as $n \rightarrow \infty$.

THEOREM 6. Let $\left(s_{n}\right)$ be a convergent sequence. If the condition of Property 3 is satisfied, then for all $k \geq 0$,

$$
E_{k+1}^{(n)}-s=o\left(E_{k}^{(n)}-s\right) \text { as } n \rightarrow \infty \Leftrightarrow \frac{E_{k}^{(n+1)}-s}{E_{k}^{(n)}-s} \underset{n \rightarrow \infty}{\longrightarrow} \lambda_{k+1} .
$$

Proof. We have

$$
\frac{E_{k+1}^{(n)}-s}{E_{k}^{(n)}-s}=\frac{\frac{g_{k, k+1}^{(n+1)}}{g_{k, k+1}^{(n)}}-\frac{E_{k}^{(n+1)}-s}{E_{k}^{(n)}-s}}{\frac{g_{k, k+1}^{(n+1)}}{g_{k, k+1}^{(n)}}-1} .
$$


From Property 4, we get

$$
\frac{g_{k, k+1}^{(n+1)}}{g_{k, k+1}^{(n)}} \underset{n \rightarrow \infty}{\longrightarrow} \lambda_{k+1}
$$

From (20), (21) we deduce that

$$
E_{k+1}^{(n)}-s=o\left(E_{k}^{(n)}-s\right) \text { as } n \rightarrow \infty \Leftrightarrow \frac{E_{k}^{(n+1)}-s}{E_{k}^{(n)}-s} \underset{n \rightarrow \infty}{\longrightarrow} \lambda_{k+1} .
$$

Rem ark. Since $\lambda_{k+1} \neq 0$, it follows that $E_{k+1}^{(n)}-s=o\left(E_{k}^{(n)}-s\right)$ as $n \rightarrow \infty$ iff $E_{k+1}^{(n)}-s=o\left(E_{k}^{(n+1)}-s\right)$ as $n \rightarrow \infty$.

Let us now assume that the sequences $\left(\lambda_{n}\right),\left(\theta_{n}\right)$ of the assumption $\left(\mathrm{H}_{2}\right)$ satisfy

$\left(\mathrm{H}_{3}\right) \quad \forall n \geq 1,0<\left|\lambda_{n}\right|<1$;

$\left(\mathrm{H}_{4}\right) \quad \forall n>m$, either $\left|\lambda_{n}\right|<\left|\lambda_{m}\right|$ or $\lambda_{n}=\lambda_{m}$ and $\theta_{n}<\theta_{m}$.

PROPERTY 5. If the auxiliary sequences $\left(g_{i}(n)\right)$ satisfy $\left(\mathrm{H}_{2}\right)$ and if $\left(\mathrm{H}_{3}\right)$, $\left(\mathrm{H}_{4}\right)$ are satisfied, then for all $k \geq 0$ and $i>k, g_{k, i}^{(n)}=o(1)$ and $g_{k, i+1}^{(n)}=$ $o\left(g_{k, i}^{(n)}\right)$ as $n \rightarrow \infty$.

Proof. Let $k \geq 0$ and $i>k$. From Property 3 , we get $g_{k, i}^{(n)} \approx$ $\lambda_{i}^{n} n^{\theta_{k, i}}\left(a_{k, i, 0}+o(1)\right)$ as $n \rightarrow \infty$, with $a_{k, i, 0} \neq 0$. Since $\left|\lambda_{i}\right|<1$, it follows that $g_{k, i}^{(n)}=o(1)$ as $n \rightarrow \infty$.

From Property 3, we obtain

$$
\frac{g_{k, i+1}^{(n)}}{g_{k, i}^{(n)}}=\left(\frac{\lambda_{i+1}}{\lambda_{i}}\right)^{n} n^{\theta_{k, i+1}-\theta_{k, i}}\left(\frac{a_{k, i+1,0}}{a_{k, i, 0}}+o(1)\right) \quad \text { as } n \rightarrow \infty .
$$

If $\left|\lambda_{i+1}\right|<\left|\lambda_{i}\right|$, then $g_{k, i+1}^{(n)}=o\left(g_{k, i}^{(n)}\right)$ as $n \rightarrow \infty$. If $\lambda_{i+1}=\lambda_{i}$, then $\theta_{i+1}<\theta_{i}$, and from Property 2 we get $\theta_{k, i+1}<\theta_{k, i}$, thus $g_{k, i+1}^{(n)}=o\left(g_{k, i}^{(n)}\right)$ as $n \rightarrow \infty$.

Remark. For each $k \geq 0,\left(g_{k, k+1}, g_{k, k+2}, \ldots, g_{k, i}, \ldots\right)$ is an asymptotic sequence.

Lemma 5. Let $\left(s_{n}\right)$ be a convergent sequence. If the conditions of Property 5 are satisfied and

$$
s_{n}-s \approx a_{1} g_{1}(n)+a_{2} g_{2}(n)+\ldots+a_{i} g_{i}(n)+\ldots,
$$

then for all $k \geq 0$,

$$
E_{k}^{(n)}-s \approx a_{k+1} g_{k, k+1}^{(n)}+\ldots+a_{i} g_{k, i}^{(n)}+\ldots
$$


Pro of (by induction on $k$ ). For $k=0$, this is obvious. Assume that

$$
E_{k-1}^{(n)}-s \approx a_{k} g_{k-1, k}^{(n)}+\ldots+a_{i} g_{k-1, i}^{(n)}+\ldots
$$

We shall prove that for all $p>k$,

$$
E_{k}^{(n)}-s=a_{k+1} g_{k, k+1}^{(n)}+\ldots+a_{p} g_{k, p}^{(n)}+o\left(g_{k, p}^{(n)}\right) \quad \text { as } n \rightarrow \infty .
$$

Let $p>k$. From the induction assumption we get

$$
E_{k-1}^{(n)}-s=a_{k} g_{k-1, k}^{(n)}+\ldots+a_{p} g_{k-1, p}^{(n)}+r_{n}
$$

with

$$
r_{n}=o\left(g_{k-1, p}^{(n)}\right) \quad \text { as } n \rightarrow \infty .
$$

We have

$$
E_{k}^{(n)}=\frac{g_{k-1, k}^{(n+1)} E_{k-1}^{(n)}-g_{k-1, k}^{(n)} E_{k-1}^{(n+1)}}{g_{k-1, k}^{(n+1)}-g_{k-1, k}^{(n)}} .
$$

From (22)-(24) we deduce that

$$
E_{k}^{(n)}=s+\sum_{i=k+1}^{p} a_{i} g_{k, i}^{(n)}+d_{n} \quad \text { with } \quad d_{n}=\frac{g_{k-1, k}^{(n+1)} r_{n}-g_{k-1, k}^{(n)} r_{n+1}}{g_{k-1, k}^{(n+1)}-g_{k-1, k}^{(n)}}
$$

We shall prove that $d_{n}=o\left(g_{k, p}^{(n)}\right)$ as $n \rightarrow \infty$. If $a_{i}=0$ for all $i>p$, then $d_{n}=0$. Assume that the coefficients $a_{i}, i>p$, are not all 0 . Let $i_{0}>p$ be the smallest index such that $a_{i_{0}} \neq 0$. We have $r_{n}=g_{k-1, i_{0}}^{(n)}\left(a_{i_{0}}+o(1)\right)$ as $n \rightarrow \infty$, and so

$$
\frac{r_{n+1}}{r_{n}}=\lambda_{i_{0}}\left(1+\frac{a}{n}+o\left(\frac{1}{n}\right)\right) \quad \text { as } n \rightarrow \infty .
$$

We have

$$
\frac{d_{n}}{g_{k, p}^{(n)}}=\frac{\frac{g_{k-1, k}^{(n+1)}}{g_{k-1, k}^{(n)}}-\frac{r_{n+1}}{r_{n}}}{\frac{g_{k-1, k}^{(n+1)}}{g_{k-1, k}^{(n)}}-\frac{g_{k-1, p}^{(n+1)}}{g_{k-1, p}^{(n)}}} \cdot \frac{r_{n}}{g_{k-1, p}^{(n)}} .
$$

From Property 3, we get

$$
\begin{array}{ll}
\frac{g_{k-1, k}^{(n+1)}}{g_{k-1, k}^{(n)}}=\lambda_{k}\left(1+\frac{\theta_{k-1, k}}{n}+o\left(\frac{1}{n}\right)\right) & \text { as } n \rightarrow \infty ; \\
\frac{g_{k-1, p}^{(n+1)}}{g_{k-1, p}^{(n)}}=\lambda_{p}\left(1+\frac{\theta_{k-1, p}}{n}+o\left(\frac{1}{n}\right)\right) & \text { as } n \rightarrow \infty .
\end{array}
$$


If $\lambda_{k} \neq \lambda_{p}$, then from (23), (25)-(28) we get $d_{n}=o\left(g_{k, p}^{(n)}\right)$ as $n \rightarrow \infty$. Assume that $\lambda_{k}=\lambda_{p}$. From Property 2 we deduce that $\theta_{k-1, k} \neq \theta_{k-1, p}$. Then, from (25)-(28) we obtain

$$
\frac{d_{n}}{g_{k, p}^{(n)}}=\frac{\left(\lambda_{k}-\lambda_{i_{0}}\right)+\frac{\lambda_{k} \theta_{k-1, k}-\lambda_{i_{0}} a}{n}+o\left(\frac{1}{n}\right)}{\lambda_{k}\left(\theta_{k-1, k}-\theta_{k-1, p}\right)+o(1)} \cdot \frac{n r_{n}}{g_{k-1, p}^{(n)}} \quad \text { as } n \rightarrow \infty
$$

If $\lambda_{k}=\lambda_{i_{0}}$, then from (23), (29) we get $d_{n}=o\left(g_{k, p}^{(n)}\right)$ as $n \rightarrow \infty$.

Assume that $\lambda_{k} \neq \lambda_{i_{0}}$. We have $n r_{n}=\lambda_{i_{0}}^{n} n^{1+\theta_{k-1, i_{0}}}\left(a_{i_{0}} a_{k-1, i_{0}, 0}+o(1)\right)$ and $g_{k-1, p}^{(n)}=\lambda_{p}^{n} n^{\theta_{k-1, p}}\left(a_{k-1, p, 0}+o(1)\right)$ as $n \rightarrow \infty$. Thus

$$
n r_{n}=o\left(g_{k-1, p}^{(n)}\right) \quad \text { as } n \rightarrow \infty \text {. }
$$

The relations (29), (30) show that $d_{n}=o\left(g_{k, p}^{(n)}\right)$ as $n \rightarrow \infty$. Finally,

$$
E_{k}^{(n)}=s+\sum_{i=k+1}^{p} a_{i} g_{k, i}^{(n)}+o\left(g_{k, p}^{(n)}\right) \quad \text { as } n \rightarrow \infty .
$$

THEOREM 7. Let $\left(s_{n}\right)$ be a convergent sequence. If $\left(\mathrm{H}_{2}\right)-\left(\mathrm{H}_{4}\right)$ are satisfied and

$$
s_{n}-s \approx a_{1} g_{1}(n)+a_{2} g_{2}(n)+\ldots+a_{i} g_{i}(n)+\ldots,
$$

then for all $k \geq 1$,

(i) if $a_{i}=0$ for all $i>k$, then $E_{k}^{(n)}=s$;

(ii) if $a_{k+1} \neq 0$, then there exists $b_{k} \neq 0$ such that $E_{k}^{(n)}-s=\lambda_{k+1}^{n} n^{\theta_{k, k+1}}$ $\times\left(b_{k+1}+o(1)\right)$ as $n \rightarrow \infty$;

(iii) if $a_{k+1}=0$, then $E_{k}^{(n)}-s=o\left(\lambda_{k+1}^{n} n^{\theta_{k, k+1}}\right)$ as $n \rightarrow \infty$.

P r o of. The results follow from Property 3 and from Lemma 5.

Remark. If $a_{i} \neq 0$ for all $i \geq 1$, then the $E$-algorithm is effective on $\left(s_{n}\right)$.

Let $0<|\lambda|<1$. Assume that the auxiliary sequences $\left(g_{i}(n)\right)$ satisfy $\left(\mathrm{H}_{2}\right)$ and

$\left(\mathrm{H}_{5}\right) \quad \forall i \geq 1, \lambda_{i}=\lambda$, and $\theta_{1}>\theta_{2}>\ldots>\theta_{i}>\theta_{i+1}>\ldots$

One can easily check that $\theta_{k, i}=\theta_{i}-k$ for all $k \geq 1$ and $i>k$.

An immediate consequence of Theorem 7 is

Corollary 1. If $s_{n}-s \approx a_{1} g_{1}(n)+\ldots+a_{i} g_{i}(n)+\ldots$ then for all $k \geq 1$, either

- $\exists n_{0}, \forall n \geq n_{0}, E_{k}^{(n)}=s$, or 
- $E_{k}^{(n)}-s=\lambda^{n} n^{\theta_{k+1}-k}\left(b_{k}+o(1)\right)$ as $n \rightarrow \infty$, with $b_{k} \neq 0$ if $a_{k+1} \neq 0$, or

- $E_{k}^{(n)}-s=o\left(\lambda^{n} n^{\theta_{k+1}-k}\right)$ as $n \rightarrow \infty$ if $a_{k+1}=0$.

Theorem 8. Let $k \geq 1$. Let $\left(s_{n}\right)$ be a convergent sequence. Assume that the conditions $\left(\mathrm{H}_{2}\right),\left(\mathrm{H}_{5}\right)$ are satisfied. If

$$
E_{k-1}^{(n)}-s \approx \lambda^{n} n^{\alpha}\left(b_{0}+\frac{b_{1}}{n^{\alpha_{1}}}+\ldots+\frac{b_{i}}{n^{\alpha_{i}}}+\ldots\right)
$$

with $b_{0} \neq 0$ and $0<\alpha_{1}<\alpha_{2}<\ldots<\alpha_{i}<\ldots$, then

$$
E_{k}^{(n)}-s \approx \lambda^{n} n^{\beta}\left(c_{0}+\frac{c_{1}}{n^{\beta_{1}}}+\ldots+\frac{c_{i}}{n^{\beta_{i}}}+\ldots\right)
$$

with $\beta \leq \alpha-1, c_{0} \neq 0$, and $0<\beta_{1}<\beta_{2}<\ldots<\beta_{i}<\ldots$

Proof. We have

$$
E_{k}^{(n)}-s=\frac{\frac{g_{k-1, k}^{(n+1)}}{g_{k-1, k}^{(n)}}-\frac{E_{k-1}^{(n+1)}-s}{E_{k-1}^{(n)}-s}}{\frac{g_{k-1, k}^{(n+1)}}{g_{k-1, k}^{(n)}}-1}\left(E_{k-1}^{(n)}-s\right) .
$$

From Property 3, we get

$$
\frac{g_{k-1, k}^{(n+1)}}{g_{k-1, k}^{(n)}} \approx \lambda+\frac{d_{1}}{n^{\gamma_{1}}}+\frac{d_{2}}{n^{\gamma_{2}}}+\ldots+\frac{d_{i}}{n^{\gamma_{i}}}+\ldots,
$$

where $1 \leq \gamma_{1}<\gamma_{2}<\ldots<\gamma_{i}<\ldots$ Moreover, we have

$$
\frac{E_{k-1}^{(n+1)}-s}{E_{k-1}^{(n)}-s} \approx \lambda+\frac{e_{1}}{n^{t_{1}}}+\frac{e_{2}}{n^{t_{2}}}+\ldots+\frac{e_{i}}{n^{t_{i}}}+\ldots,
$$

where $1 \leq t_{1}<t_{2}<\ldots<t_{i}<\ldots$ From (32), (33) we obtain

$$
\frac{g_{k-1, k}^{(n+1)}}{g_{k-1, k}^{(n)}}-\frac{E_{k-1}^{(n+1)}-s}{E_{k-1}^{(n)}-s} \approx \frac{f_{1}}{n^{p_{1}}}+\ldots+\frac{f_{j}}{n^{p_{j}}}+\ldots
$$

with $f_{1} \neq 0$ and $1 \leq p_{1}<p_{2}<\ldots<p_{j}<\ldots$ Furthermore, we have

$$
E_{k-1}^{(n)}-s \approx \lambda^{n} n^{\alpha}\left(b_{0}+\frac{b_{1}}{n^{\alpha_{1}}}+\ldots+\frac{b_{i}}{n^{\alpha_{i}}}+\ldots\right) .
$$

From (31), (32), (34), (35) we deduce that

$$
E_{k}^{(n)}-s \approx \lambda^{n} n^{\beta}\left(c_{0}+\frac{c_{1}}{n^{\beta_{1}}}+\ldots+\frac{c_{j}}{n^{\beta_{j}}}+\ldots\right),
$$

where $\beta=\alpha-p_{1}, c_{0}=f_{1} b_{0} /(\lambda-1)$, and $0<\beta_{1}<\beta_{2}<\ldots<\beta_{i}<\ldots$ 
An immediate consequence of Theorem 8 is

Corollary 2. Let $\left(s_{n}\right)$ be a convergent sequence. If the conditions $\left(\mathrm{H}_{2}\right)$, $\left(\mathrm{H}_{5}\right)$ are satisfied and

$$
s_{n}-s \approx \lambda^{n} n^{\alpha_{0}}\left(a_{0}+\frac{a_{1}}{n^{\alpha_{0,1}}}+\ldots+\frac{a_{i}}{n^{\alpha_{0, i}}}+\ldots\right)
$$

with $a_{0} \neq 0$ and $0<\alpha_{0,1}<\alpha_{0,2}<\ldots<\alpha_{0, i}<\ldots$, then there exists a strictly decreasing sequence $\left(\alpha_{k}\right)$ such that $\alpha_{k} \leq \alpha_{0}-k$ for all $k \geq 1$, and

$$
E_{k}^{(n)}-s \approx \lambda^{n} n^{\alpha_{k}}\left(a_{k, 0}+\frac{a_{k, 1}}{n^{\alpha_{k, 1}}}+\ldots+\frac{a_{k, i}}{n^{\alpha_{k, i}}}+\ldots\right)
$$

with $a_{k, 0} \neq 0$ and $0<\alpha_{k, 1}<\ldots<\alpha_{k, i}<\ldots$

Remark. The $E$-algorithm is effective on $\left(s_{n}\right)$.

It was shown by Brezinski [2] that the $E$-algorithm includes the following sequence transformations: Shanks transformation $\left(g_{i}(n)=\Delta s_{n+i-1}\right)[1,13]$, the process $p\left(g_{1}(n)=\lambda^{n} n^{\theta}, \theta \in \mathbb{R}\right.$, and $g_{i}(n)=\Delta s_{n+i-2}$ for $\left.i \geq 2\right)$, Levin's transformations $\left(g_{i}(n)=g(n) \Delta s_{n-1} / n^{i-1}\right.$, with $g(n)=1(\operatorname{resp} . g(n)=n$, $\left.g(n)=\Delta s_{n} \Delta s_{n-1} / \Delta^{2} s_{n-1}\right)$ ) for the transformation $T$ (resp. $U, V$ ) [9].

If $\left(s_{n}\right)$ satisfies $(36)$, then for each of these transformations, one can easily prove that for all $i \geq 1$,

$$
g_{i}(n) \approx \lambda^{n} n^{\theta_{i}}\left(b_{i, 0}+\frac{b_{i, 1}}{n^{\beta_{i, 1}}}+\ldots+\frac{b_{i, j}}{n^{\beta_{i, j}}}+\ldots\right) .
$$

Then, from the preceding remark, we deduce

Corollary 3. If $\left(s_{n}\right)$ satisfies (36), then the Shanks transformation, the process $p$, and Levin's transformations $(T, U$, and $V)$ are effective on $\left(s_{n}\right)$.

Let us end this subsection with

THEOREM 9. If $\left(s_{n}\right)$ satisfies (36), then the algorithm

$$
\begin{aligned}
E_{0}^{(n)} & =s_{n}, & & n \geq 0, \\
E_{k+1}^{(n)} & =\frac{\lambda E_{k}^{(n)}-E_{k}^{(n+1)}}{\lambda-1}, & & k \geq 1,
\end{aligned}
$$

is effective on $\left(s_{n}\right)$.

Proof. By induction on $k$, we prove that for all $k \geq 0$,

$$
E_{k}^{(n)}-s \approx \lambda^{n} n^{\alpha_{k}}\left(a_{k, 0}+\frac{a_{k, 1}}{n^{\alpha_{k, 1}}}+\ldots+\frac{a_{k, i}}{n^{\alpha_{k, i}}}+\ldots\right)
$$

with $\alpha_{k}<\alpha_{k-1}$ and $0<\alpha_{k, 1}<\alpha_{k, 2}<\ldots<\alpha_{k, i}<\ldots$ Then, the result follows immediately. 
2.2. Logarithmic convergence. In this section, the auxiliary sequences $\left(g_{i}(n)\right)$ of the $E$-algorithm are such that

$$
\forall i \geq 1, \quad g_{i}(n) \approx n^{\theta_{i}}\left(a_{i, 0}+\frac{a_{i, 1}}{n^{\alpha_{i, 1}}}+\ldots+\frac{a_{i, j}}{n^{\alpha_{i, j}}}+\ldots\right)
$$

with $a_{i, 0} \neq 0$ and $0>\theta_{1}>\theta_{2}>\ldots>\theta_{i}>\ldots$

Lemma 6. For all $k \geq 0$ and $i>k$,

$$
g_{k, i}^{(n)} \approx n^{\theta_{i}}\left(a_{k, i, 0}+\frac{a_{k, i, 1}}{n^{\alpha_{k, i, 1}}}+\ldots+\frac{a_{k, i, j}}{n^{\alpha_{k, i, j}}}+\ldots\right),
$$

where $a_{k, i, 0} \neq 0$ and $0<\alpha_{k, i, 1}<\alpha_{k, i, 2}<\ldots<\alpha_{k, i, j}<\ldots$

Proof. By induction on $k$.

By using Lemma 6, one can easily prove

Property 6. For all $k \geq 0$ and $i>k, g_{k, i}^{(n)}=o(1)$ and $g_{k, i+1}^{(n)}=o\left(g_{k, i}^{(n)}\right)$ as $n \rightarrow \infty$, and

$$
g_{k, i}^{(n+1)} / g_{k, i}^{(n)} \underset{n \rightarrow \infty}{\longrightarrow} 1
$$

Remark. For each $k \geq 0,\left(g_{k, k+1}, g_{k, k+2}, \ldots, g_{k, i}, \ldots\right)$ is an asymptotic sequence.

Property 7. The E-algorithm is not regular on the set of convergent sequences.

Proof. This follows from the fact that

$$
\forall k \geq 0, \quad \frac{g_{k, k+1}^{(n+1)}}{g_{k, k+1}^{(n)}} \underset{n \rightarrow \infty}{\longrightarrow} 1 .
$$

LEMma 7 . If $s_{n}-s \approx a_{1} g_{1}(n)+\ldots+a_{i} g_{i}(n)+\ldots$, then for all $k \geq 0$,

$$
E_{k}^{(n)}-s \approx a_{k+1} g_{k, k+1}^{(n)}+\ldots+a_{i} g_{k, i}^{(n)}+\ldots
$$

Proof. By induction on $k$.

Using Lemmas 6 and 7, one can easily prove

THEOREM 10. If $s_{n}-s \approx a_{1} g_{1}(n)+\ldots+a_{i} g_{i}(n)+\ldots$, then for all $k \geq 1$, either

- $\exists n_{0}, \forall n \geq n_{0}, E_{k}^{(n)}=s$, or

- $E_{k}^{(n)}-s=n^{\theta_{k+1}}\left(b_{k}+o(1)\right)$ as $n \rightarrow \infty$, with $b_{k} \neq 0$ if $a_{k+1} \neq 0$, or

- $E_{k}^{(n)}-s=o\left(n^{\theta_{k+1}}\right)$ as $n \rightarrow \infty$ if $a_{k+1}=0$. 
Remark. If $a_{i} \neq 0$ for all $i \geq 1$, then the $E$-algorithm is effective on $\left(s_{n}\right)$.

2.3. Numerical instability. Consider the E-algorithm of the preceding subsections. We have for all $i \geq 1$,

$$
g_{i}(n) \approx \lambda_{i}^{n} n^{\theta_{i}}\left(a_{i, 0}+\frac{a_{i, 1}}{n^{\alpha_{i, 1}}}+\ldots+\frac{a_{i, j}}{n^{\alpha_{i, j}}}+\ldots\right) \text { and } \frac{g_{i-1, i}^{(n+1)}}{g_{i-1, i}^{(n)}} \underset{n \rightarrow \infty}{\longrightarrow} \lambda_{i}
$$

When the numbers $\lambda_{i}$ are close to 1 , the $E$-algorithm is numerically unstable. Then, in practice, the properties of convergence acceleration are lost, and a good approximate value of the limit of $\left(s_{n}\right)$ cannot be computed. In order to avoid numerical instability, we propose to use some subsequences of the auxiliary sequences $\left(g_{i}(n)\right), i \geq 1$.

For example, set $h_{i}(n)=g_{i}\left(2^{n}\right), i \geq 1$. Then for all $i \geq 1$,

$$
h_{i}(n) \approx \lambda_{i}^{2^{n}} 2^{n \theta_{i}}\left(a_{i, 0}+a_{i, 1}\left(\frac{1}{2^{\alpha_{i, 1}}}\right)^{n}+\ldots\right) .
$$

If $0<\left|\lambda_{i}\right|<1$ for all $i \geq 1$, then

$$
\forall i \geq 1, \quad \frac{h_{i}(n+1)}{h_{i}(n)} \underset{n \rightarrow \infty}{\longrightarrow} 0 .
$$

If $\lambda_{i}=1$ for all $i \geq 1$, then

$$
\forall i \geq 1, \quad \frac{h_{i}(n+1)}{h_{i}(n)} \underset{n \rightarrow \infty}{\longrightarrow} 2^{\theta_{i}}
$$

with $0>\theta_{1}>\theta_{2}>\ldots>\theta_{i}>\ldots$ Consequently, the $E$-algorithm with $\left(h_{i}(n)\right)$ as auxiliary sequences is more stable than the $E$-algorithm with $\left(g_{i}(n)\right)$ as auxiliary sequences.

Let us mention that the $E$-algorithm with $\left(h_{i}(n)\right)$ as auxiliary sequences must be applied to the subsequence $\left(s_{2^{n}}\right)$ of $\left(s_{n}\right)$.

Let us now compare the two algorithms in terms of the number of arithmetical operations.

It was shown by Brezinski [2] that the computation of $E_{k}^{0}$ from $s_{0}, \ldots, s_{k}$ by the $E$-algorithm needs approximately $\frac{5}{3} k^{3}$ arithmetical operations. Consequently, for computing an approximate value of the limit of $\left(s_{n}\right)$ from $s_{0}, \ldots, s_{2^{n}}$, the $E$-algorithm with $\left(g_{i}(n)\right)$ as auxiliary sequences needs $O\left(2^{3 n}\right)$, and the $E$-algorithm with $\left(h_{i}(n)\right)$ as auxiliary sequences applied to $\left(s_{2^{n}}\right)$ needs only $O\left(n^{3}\right)$ arithmetical operations.

Some numerical examples illustrating this technique will be given in the following section. 


\section{Applications}

3.1. Summation of series. Consider the power series $s(x)=\sum_{n=0}^{\infty} a_{n} x^{n}$, where

$$
a_{n} \approx n^{\theta}\left(\alpha_{0}+\frac{\alpha_{1}}{n}+\ldots+\frac{\alpha_{i}}{n^{i}}+\ldots\right) \quad \text { with } \alpha_{0} \neq 0 .
$$

Let $s_{n}(x)=\sum_{k=0}^{n} a_{k} x^{k}$ for $n \geq 0$. From a result of Wimp ([14], p. 19), we deduce the following.

If $|x| \leq 1$ and $x \neq 1$, then

$$
s_{n}(x)-s(x) \approx x^{n} n^{\theta}\left(\beta_{0}+\frac{\beta_{1}}{n}+\ldots\right) \quad \text { with } \beta_{0} \neq 0 .
$$

If $x=1$ and $\theta<-1$, then

$$
s_{n}(1)-s(1) \approx n^{\theta+1}\left(\gamma_{0}+\frac{\gamma_{1}}{n}+\ldots\right) \quad \text { with } \gamma_{0} \neq 0 .
$$

1) Setting $g_{i}(n)=x^{n} n^{\theta-i+1}$ for $i \geq 1$, the auxiliary sequences satisfy the conditions of Section 2. Then the $E$-algorithm is effective on $\left(s_{n}(x)\right)$.

2 ) Let us now consider the subsequence $\left(s_{2^{n}}(x)\right)$ of $\left(s_{n}(x)\right)$. We have

$$
s_{2^{n}}(x)-s(x) \approx\left(x^{2^{n}}\right) 2^{n \theta}\left(\beta_{0}+\frac{\beta_{1}}{2^{n}}+\ldots+\frac{\beta_{i}}{\left(2^{i}\right)^{n}}+\ldots\right) .
$$

If $0<|x|<1$, then we can apply the results of Section 1 for the $E$-algorithm with the auxiliary sequences

$$
h_{i}(n)=\left(x^{2^{n}}\right) 2^{n \theta} b_{i}^{n} \quad \text { and } \quad b_{i}=1 / 2^{i-1} .
$$

$3)$ Let $0<|x|<1, x \neq 1$. Then $\left(s_{n}(x)\right)$ satisfies (36), and from Corollary 3 and Theorem 9 we deduce that the Shanks transformation, the process $p$, Levin's transformations, and the sequence transformation given in Theorem 9 are all effective on $\left(s_{n}\right)$.

Let us now give some numerical examples. We begin by the following linearly convergent series:

$$
\sum_{n=0}^{\infty}(n+1) x^{n}=\frac{1}{(x-1)^{2}}, \quad x=0.9,0.99,0.999 .
$$

The results obtained by applying the $E$-algorithm to $\left(s_{n}\right)$ (resp. $\left(s_{2^{n}}\right)$ ) with $g_{i}(n)=x^{n} n^{\theta-i+1}$ (resp. $h_{i}(n)=g_{i}\left(2^{n}\right)$ ) as auxiliary sequences are summarized in Table 1 (resp. Table 2), where we indicate, at each step $n$, the number of exact digits of $E_{n}^{0}$. 


\begin{tabular}{|c|c|c|c|}
\hline$n$ & .9 & .99 & .999 \\
\hline 2 & 13 & 13 & 11 \\
\hline 4 & 13 & 12 & 8 \\
\hline 8 & 12 & 12 & 10 \\
\hline 16 & 9 & 8 & 5 \\
\hline 32 & 6 & 0 & 0 \\
\hline
\end{tabular}

\begin{tabular}{rrrr}
\multicolumn{4}{c}{ T A B LE } \\
\hline$n$ & .9 & .99 & .999 \\
\hline 1 & 0 & 0 & 0 \\
2 & 14 & 13 & 10 \\
3 & 15 & 13 & 11 \\
4 & 15 & 14 & 10 \\
5 & 15 & 15 & 11 \\
\hline
\end{tabular}

The comparison of Tables 1 and 2 shows that the $E$-algorithm with $\left(h_{i}(n)\right)$ as auxiliary sequences is more stable than the $E$-algorithm with $\left(g_{i}(n)\right)$ as auxiliary sequences.

Let us now consider the following logarithmic convergent series [4]:

1) $\sum_{n=1}^{\infty} \frac{1}{n^{2}}=\frac{\pi^{2}}{6}$

2) $\sum_{n=1}^{\infty} \frac{n^{4}+n^{2}+1}{n^{2}\left(1+n^{4}\right)} \cong 2.223411646515$,

3) $\sum_{n=1}^{\infty}\left(n+e^{1 / n}\right)^{-\sqrt{2}} \cong 1.71379673554030$,

4) $\sum_{n=1}^{\infty} \log \left(\frac{n+1}{n}\right) \log \left(\frac{n+2}{n+1}\right) \cong .68472478856$,

5) $1+\sum_{n=0}^{\infty}\left(\frac{1}{n+1}+\log \left(\frac{n}{n+1}\right)\right) \cong .57721566490153286$.

The results obtained by applying the $E$-algorithm to $\left(s_{n}\right)$ with $\left(g_{i}(n)\right)$, $i \geq 1$, as auxiliary sequences are given in Table 3 .

T A B LE 3

\begin{tabular}{rrrrrrrrrrr}
\hline$n$ & \multicolumn{3}{c}{$1)$} & \multicolumn{3}{c}{$2)$} & \multicolumn{3}{c}{$3)$} & \multicolumn{3}{c}{$4)$} \\
\hline & $s_{n}$ & $E_{n}^{0}$ & $s_{n}$ & $E_{n}^{0}$ & $s_{n}$ & $E_{n}^{0}$ & $s_{n}$ & $E_{n}^{0}$ & $s_{n}$ & $E_{n}^{0}$ \\
\hline 2 & 1 & 1 & 0 & 1 & 0 & 1 & 1 & 2 & 1 & 3 \\
4 & 1 & 3 & 0 & 2 & 0 & 2 & 1 & 4 & 1 & 4 \\
8 & 1 & 6 & 1 & 5 & 0 & 5 & 1 & 8 & 1 & 10 \\
12 & 1 & 11 & 1 & 9 & 0 & 9 & 1 & 10 & 1 & 10 \\
14 & 1 & 11 & 1 & 9 & 0 & 9 & 2 & 10 & 1 & 11 \\
16 & 1 & 9 & 1 & 8 & 0 & 8 & 2 & 9 & 1 & 9 \\
32 & 2 & 4 & 1 & 5 & 1 & 3 & 2 & 4 & 2 & 5 \\
40 & 2 & 4 & 1 & 3 & 1 & 3 & 2 & 4 & 2 & 4 \\
50 & 2 & 2 & 2 & 4 & 1 & 3 & 2 & 3 & 2 & 3 \\
\hline
\end{tabular}

Applying the $E$-algorithm to $\left(s_{2^{n}}\right)$, with $\left(h_{i}(n)\right)$ as auxiliary sequences, 
we obtain the results given in Table 4 .

T A B L E 4

\begin{tabular}{rrrrrrrrrrrr}
\hline$n$ & \multicolumn{3}{c}{$1)$} & \multicolumn{2}{c}{$2)$} & \multicolumn{3}{c}{$3)$} & \multicolumn{2}{c}{$4)$} & \multicolumn{2}{c}{$5)$} \\
\hline & $s_{2^{n}}$ & $E_{n}^{0}$ & $s_{2^{n}}$ & $E_{n}^{0}$ & $s_{2^{n}}$ & $E_{n}^{0}$ & $s_{2^{n}}$ & $E_{n}^{0}$ & $s_{2^{n}}$ & $E_{n}^{0}$ \\
\hline 1 & 1 & 1 & 0 & 1 & 0 & 0 & 1 & 1 & 1 & 1 \\
2 & 1 & 2 & 0 & 2 & 0 & 1 & 1 & 2 & 1 & 2 \\
3 & 1 & 4 & 1 & 3 & 0 & 1 & 1 & 2 & 1 & 3 \\
4 & 1 & 6 & 1 & 4 & 1 & 3 & 2 & 4 & 1 & 4 \\
5 & 2 & 7 & 1 & 5 & 1 & 4 & 2 & 4 & 2 & 5 \\
6 & 2 & 9 & 2 & 9 & 1 & 6 & 2 & 7 & 2 & 7 \\
7 & 2 & 11 & 2 & 10 & 1 & 8 & 2 & 8 & 2 & 9 \\
8 & 3 & 13 & 2 & 12 & 1 & 10 & 3 & 10 & 3 & 12 \\
9 & 3 & 15 & 3 & 14 & 1 & 11 & 3 & 12 & 3 & 15 \\
\hline
\end{tabular}

The results of Tables 3 and 4 show that the $E$-algorithm with $\left(h_{i}(n)\right)$ as auxiliary sequences is more effective than the $E$-algorithm with the auxiliary sequences $\left(g_{i}(n)\right)$.

3.2. Numerical quadrature. The numerical computation of the integral $s=\int_{0}^{1} f(x) d x$ often leads to an asymptotic expansion of the form

$$
T(h)-s \approx \sum_{j=1}^{\infty} \sum_{i=0}^{m_{j}} a_{j, i}(\log (h))^{i} h^{\gamma_{j}}
$$

with $a_{j, m_{j}} \neq 0, m_{j} \in \mathbb{N}, 0<\gamma_{1}<\gamma_{2}<\ldots<\gamma_{j}<\ldots$, where $T(h)$ is an approximate value of $s$ obtained by some quadrature formulae with steplength $h([0,1]$ is divided into $1 / h$ subintervals of length $h$; see $[5-8$, 10-12]).

Let $\sigma \geq 2, \sigma \in \mathbb{N}$. For $h_{n}=1 / \sigma^{n}$, we have

$$
T\left(h_{n}\right)-s \approx \sum_{j=1}^{\infty} \sigma^{-n \gamma_{j}} n^{m_{j}}\left(a_{j, m_{j}}+\ldots+\frac{a_{j, 1}}{n^{m_{j}-1}}+\frac{a_{j, 0}}{n^{m_{j}}}\right) .
$$

Let $\left(p_{n}\right)$ be the sequence defined by $p_{0}=0, p_{n}=n+\sum_{i=1}^{n} m_{i}$ for $n \geq 1$. Let $j \geq 1$. Set $\theta_{i}=p_{j}-i$ and $\lambda_{i}=\sigma^{-\gamma_{j}}$ for $i=1+p_{j-1}, \ldots, p_{j}$. The sequence $s_{n}=T\left(h_{n}\right)$ has the asymptotic expansion

$$
s_{n}-s \approx a_{1} g_{1}(n)+\ldots+a_{k} g_{k}(n)+\ldots \quad \text { with } g_{i}(n)=\lambda_{i}^{n} n^{\theta_{i}} \text { for } i \geq 1 .
$$

Thus, we can apply the results of Section 2 .

Let us end this section with a numerical example. We have

$$
s=\int_{0}^{1} \frac{x \log x}{x+1} d x=\frac{\pi^{2}}{12}-1 \cong-.1775329665759 .
$$


Let $T(h)$ be the approximate value of $s$ obtained by the trapezoidal rule with steplength $h$. We have

$$
T(h)-s \approx \sum_{i=2}^{\infty} h^{i}\left(a_{i, 1}+a_{i, 2} \log h\right) \quad(\text { see }[5]) .
$$

For $\sigma=2$, we have

\begin{tabular}{ccc}
\hline$n$ & $E_{0}^{n}$ & $E_{n}^{0}$ \\
\hline 2 & -.1758293422171846 & -.1775484527978632 \\
4 & -.1773979134959518 & -.1775329593577489 \\
6 & -.1775227574385099 & -.1775329674394418 \\
8 & -.1775322183192145 & -.1775329667708583 \\
\hline
\end{tabular}

Acknowledgements. I want to thank the referee for his valuable suggestions. The paper has also profited from discussions with A. Lembarki, Faculty of Semlalia.

\section{References}

[1] C. Brezinski, Algorithmes d'Accélération de la Convergence. Etude Numérique, Technip, Paris, 1978.

[2] —, A general extrapolation algorithm, Numer. Math. 35 (1980), 175-187.

[3] C. Brezinski and M. Redivo Zaglia, Extrapolation Methods, Theory and Practice, North-Holland, Amsterdam, 1991.

[4] W. F. Ford and D. A. Smith, Acceleration of linear and logarithmic convergence, SIAM J. Numer. Anal. 16 (1979), 223-240.

[5] L. Fox, Romberg integration for a class of singular integrands, Comput. J. 10 (1967), 87-93.

[6] T. Håvie, Error derivation in Romberg integration, BIT 12 (1972), 516-527.

[7] -, Generalized Neville type extrapolation schemes, ibid. 19 (1979), 204-213.

[8] D. C. Joyce, Survey of extrapolation processes in numerical analysis, SIAM Rev. 13 (1972), 435-487.

[9] D. Levin, Development of nonlinear transformations for improving convergence of sequences, Internat. J. Computer Math. 3 (1973), 371-388.

[10] J. N. Lyness, Applications of extrapolation techniques to multidimensional quadrature of some integrand functions with a singularity, J. Comput. Phys. 20 (1976), 346-364.

[11] J. N. Lyness and E. de Doncker-Kapenga, On quadrature error expansions, Part I, J. Comput. Appl. Math. 17 (1987), 131-149.

[12] J. N. Lyness and B. W. Ninham, Numerical quadrature and asymptotic expansions, Math. Comput. 21 (1967), 162-178.

[13] D. Shanks, Non-linear transformations of divergent and slowly convergent sequences, J. Math. Phys. 34 (1955), 1-42. 
[14] J. Wimp, Sequence Transformations and their Applications, Academic Press, New York, 1984.

A. Fdil

Département de Mathématiques

E.N.S. de Marrakech

B.P. S 41

40000 Marrakech, Morocco

Received on 28.5.1996;

revised version on 7.11 .1996 